\title{
Normal ranges of fetal heart rate values for healthy fetuses in Poland, as determined by ultrasound between weeks 18 and 29 of gestation
}

\author{
Oskar Sylwestrzak ${ }^{1,2}$, Aleksandra Nowakowska', Julia Murlewska², Maria Respondek-Liberska²,3 \\ 'Medical Faculty, Medical University of Lodz, Łódź, Poland \\ 2Department for Prenatal Cardiology, Polish Mother's Memorial Hospital Research Institute, Łódź, Poland \\ ${ }^{3}$ Department of Diagnosis and Prevention Fetal Malformations, Medical University of Lodz, Łódź, Poland
}

Correspondence to: Oskar Sylwestrzak, MD, Department of Prenatal Cardiology, Hospital Research Institute, Rzgowska 281/289, 93-338 Łódź, Poland,

phone: +48 422711556 ,

e-mail:

sylwestrzakoskarpatryk@ gmail.com

Copyright by the Author(s), 2021

Kardiol Pol. 2021;

79 (11): 1245-1250

DOI: 10.33963/KP.a2021.0119

Received:

March 3, 2021

Accepted:

September 22, 2021

Published online:

September 22, 2021

\author{
A B S TR A C T \\ Background: Fetal heart rate (FHR) is commonly used to assess fetal well-being.
}

Aims: The aim was to establish normal ranges of FHR during pregnancy by umbilical artery (UMB-A) Doppler analysis in a healthy Polish population.

Methods: The study was conducted in a tertiary center for fetal cardiology. Data on gestational age (GA), FHR measured on UMB-A, cardiac problems, and extracardiac problems were collected. All fetuses underwent echocardiographic examination. The inclusion criteria comprised normal fetal biometry and biophysical profile, labels of "normal heart anatomy and normal heart function", "no extracardiac malformations", and no "extracardiac anomalies".

Results: Based on the data from 258 healthy fetuses, a scatter graph with regression line giving a prognosis of normal values for FHR during pregnancy was prepared (95\% confidence interval). The regression equation for FHR, as function of GA in weeks, was found to be: FHR (beats $/$ minute) $=149-\mathrm{GA}$ according to biometry (weeks) $\times 0.22 ;(r=-0.1032 ; P=0.098) ; \mathrm{FHR}$ (beats/ $/$ minute) $=148-\mathrm{GA}$ according to the last menstrual period (weeks) $\times 0.16 ;(r=-0.0722 ; P=0.253)$. The $5^{\text {th }}, 50^{\text {th }}, 95^{\text {th }}$ percentiles, mean and standard deviation of FHR between weeks 18 and 29 of gestation were calculated.

Conclusions: We present the normal ranges for FHR measured on UMB-A for weeks 18 to 29 of gestation in healthy fetuses with normal heart function and anatomy. The obtained values may be of value to departments of obstetrics and should be considered important elements of the basic fetal ultrasound report.

Key words: fetal echocardiography, fetal heart rate, prenatal, ultrasound, umbilical artery

Kardiol Pol 2021; 79, 11: 1245-1250

\section{INTRODUCTION}

The heart is the first organ of the human body to develop in fetal life and its contractions are the first markers of live pregnancy [1]. As such, fetal heart rate (FHR) is commonly used to assess fetal well-being at the earliest stages of fetal life to the late third trimester of pregnancy. Despite its long history, FHR remains one of the most important parameters obtained during a prenatal ultrasound examination. However, there is still no consensus as to what constitutes a normal FHR.
Current guidelines suggest normal FHR ranges of 110 to $150 \mathrm{bpm}$ or 110 to $160 \mathrm{bpm}$ [2]; however, these values vary depending on various factors, such as the stage of pregnan$c y$, maternal health, and uterus conditions [3]. No recent studies have examined normal FHR assessment by ultrasound, especially in Poland; therefore, as normal ranges may depend on ethnicity or region of living [4] and considering the rapid changes in Poland over the past couple of decades, there is arguably a need to revise these FHR ranges 
WHAT'S NEW?

Fetal heart rate (FHR) is commonly used to assess fetal well-being. However, no normal ranges of FHR have been published for the Polish population, even though Poland has undergone considerable environmental and structural changes. We present the normal ranges for FHR measured on the umbilical artery in healthy fetuses with normal heart function and anatomy between weeks 18 and 29 of gestation. To identify healthy fetuses, obstetrical ultrasound examination was supplemented with fetal echocardiography. The obtained nomograms can be used by prenatal and obstetrical departments in Poland to improve FHR assessment and should be considered an important element of a basic fetal ultrasound report.

and establish nomograms, especially for the healthy population.

Various methods are used for FHR assessment; however, the standard approach is based on measuring the blood flow in the umbilical cord by Doppler ultrasound $[5,6]$. Therefore, this study aimed to establish normal ranges of FHR values for healthy fetuses in Poland during gestation, as determined by ultrasound.

\section{METHODS}

Prenatal ultrasound and echocardiographic examinations were performed on singleton fetuses in a single tertiary fetal cardiology center. Examinations were performed by specialists in fetal medicine using GE Voluson E8, GE Voluson 10 (GE Healthcare, Chicago, IL, USA), and Philips iU22 (Philips, Amsterdam, Netherlands) ultrasound equipment. Gestational age (GA) was calculated based on the last menstrual period (LMP) and fetal biometry.

All patients gave their permission for their data to be used for scientific analyses. As the present study focused on the interpretation of previously collected data, rather than performing ultrasound - echocardiographic examinations, no additional approval was required from the local Ethical Committee.

\section{Estimation of normal FHR values}

Data on gestational age at examination, FHR, cardiac problems, and extracardiac problems were collected and stored in the records at the clinic. FHR values measured on the umbilical artery (UMB-A) were calculated based on automatic presets on the ultrasound machines. The steps were as follows: (1) umbilical cord was first demonstrated; (2) to obtain the proper plane for measurement, a part of UMB-A parallel to the Doppler gate was selected; (3) the transducer was positioned to obtain the best angle between the Doppler gate and the blood flow through UMB-A. Three sets of measurements were made, and the values were averaged and recorded. Every fetus underwent an umbilical blood flow assessment with color Doppler, FHR measurement, and pulsatility index calculation. All fetuses underwent at least one echocardiographic examination.

The inclusion criteria comprised normal fetal biometry and biophysical profile, and the labeled "normal heart anatomy and normal heart function" in the database, i.e. without "extracardiac malformations" or "extracardiac anomalies". Cases with maternal diabetes, maternal Hashimoto, maternal heart problems, maternal hypertension, functional anomalies, oligo- or polyhydramnios, and a two-vessel cord or any other fetal abnormality were excluded, as well as premature deliveries. Cases with information about maternal drug use, which could have influenced FHR, were excluded. Only completed records with information about delivery and newborn conditions were included in the search (labeled in our database as "birth at term").

\section{Statistical analysis}

All statistical analyses were performed using Statistica 13.1 software. Results are expressed as mean (standard deviation [SD]), median (interquartile range [IQR]), or 95\% confidence interval $(\mathrm{Cl})$ as appropriate. The normality of distribution was tested using Shapiro-Wilk and Kolmogorov-Smirnov tests (the tests confirmed normality of distribution of FHR with gestation $P>0.05$ ). The association between dependent and independent variables was evaluated by Pearson correlation and linear regression. A $P$-value $<0.05$ was considered statistically significant. Based on the best-fit equation and the predicted standard deviation, predictive values for $5^{\text {th }}, 50^{\text {th }}$, and $95^{\text {th }}$ percentile ranges of FHR were constructed.

\section{RESULTS}

The age of the studied fetuses ranged from $\mathrm{min} .18+2$ to max. $29+5$ (weeks of gestation + days), according to fetal biometry, and from min. $18+2$ to max. $28+5$ (weeks of gestation + days), according to LMP, in singleton pregnancies. Out of a total of 763 cases studied between 2016 and 2020, 505 were excluded due to a lack of delivery data or FHR information. Therefore, 258 healthy fetuses were included in the study group.

A scatter graph with regression line giving a prognosis of normal values for FHR during pregnancy was prepared $(95 \% \mathrm{Cl}$ ) (Figures 1 and 2). The regression equation for FHR as a function of $G A$ in weeks was:

- FHR (bpm) $=149-\mathrm{GA}$ according to biometry (weeks) $\times 0.22 ;(r=-0.1032 ; P=0.098)$;

- $\quad \mathrm{FHR}(\mathrm{bpm})=148-\mathrm{GA}$ according to LMP (weeks) $\times 0.16$; $(r=-0.0722 ; P=0.253)$. 


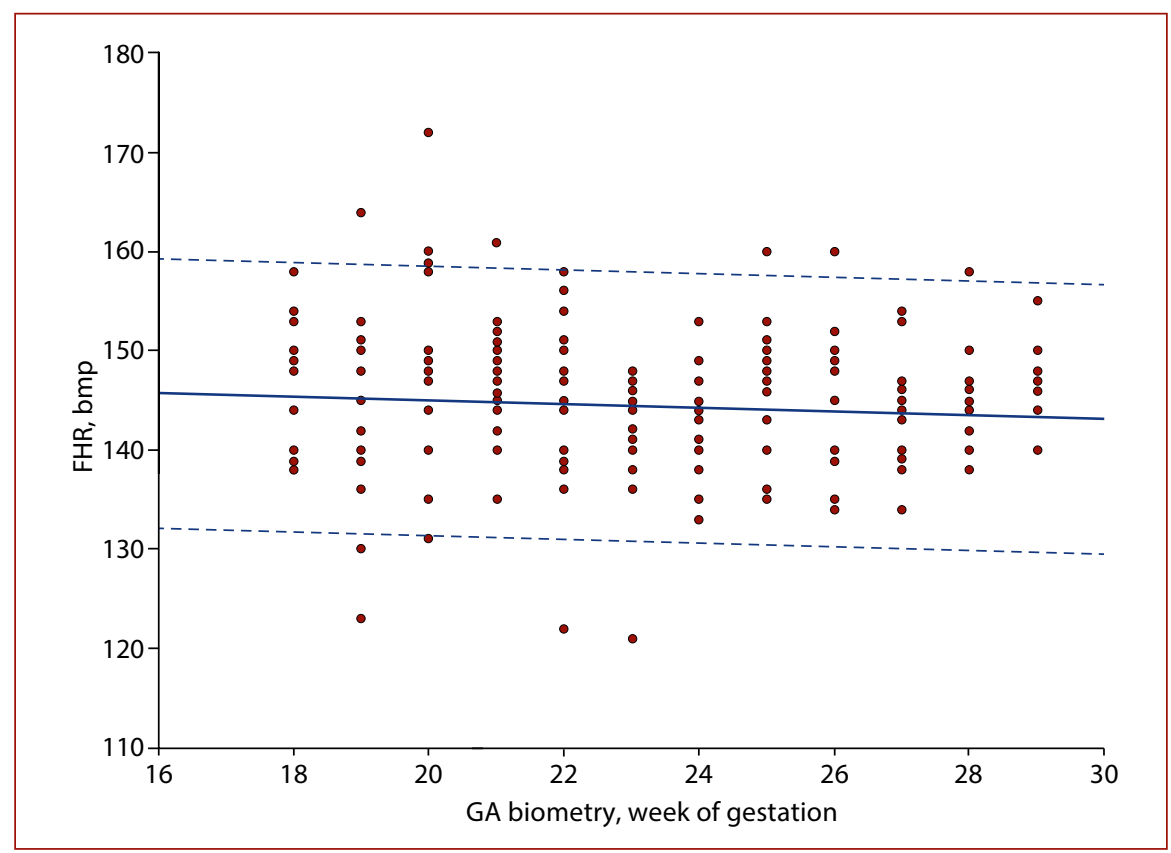

Figure 1. Scatter graph of fetal heart rate (FHR) by gestational age (GA) (biometry). The three lines represent the predicted value of mean and $95 \%$ confidence intervals

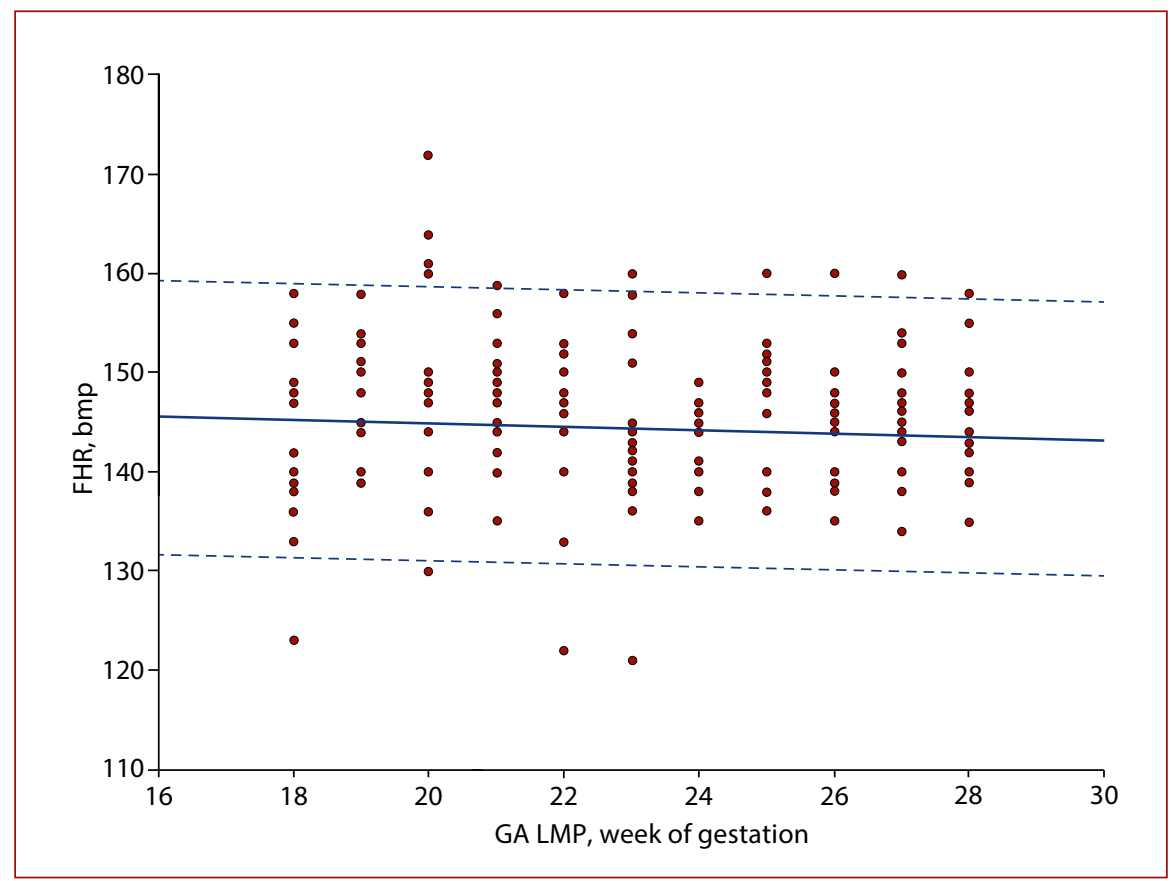

Figure 2. Scatter graph of fetal heart rate (FHR) by gestational age (GA). The three lines represent the predicted value of mean and $95 \%$ confidence intervals

Abbreviations: LMP, last menstrual period

Linear regression demonstrated no statistically significant correlation between FHR and GA.

The $5^{\text {th }}, 50^{\text {th }}, 95^{\text {th }}$ percentiles of FHR between weeks 18 and 28 (for GA according to LMP) or 29 (for GA according to biometry) of gestation were calculated. The percentiles of FHR with mean and standard deviation are given in Tables 1 and 2.

\section{DISCUSSION}

The present study examined FHR values previously obtained by ultrasound measurements of the blood flow in the UMB-A, one of the easiest prenatal assessments to perform $[7,8]$. The ultrasound beam penetrates tissues and is reflected whenever it encounters a change in acoustic impedance [9]. When reflected from a moving object, the 
Table 1. Fetal heart rate according to fetal biometry during gestation

\begin{tabular}{|c|c|c|c|c|c|}
\hline GA, week & Number of cases & Mean (SD) & $5^{\text {th }}$ percentile & $50^{\text {th }}$ percentile & $95^{\text {th }}$ percentile \\
\hline 18 & 13 & $147(6)$ & 138 & 148 & 158 \\
\hline 19 & 20 & $143(9)$ & 127 & 144 & 159 \\
\hline 20 & 27 & $146(9)$ & 135 & 145 & 160 \\
\hline 21 & 28 & $146(6)$ & 140 & 147 & 153 \\
\hline 22 & 22 & $145(8)$ & 136 & 146 & 158 \\
\hline 23 & 19 & $142(7)$ & 121 & 141 & 158 \\
\hline 24 & 20 & $141(5)$ & 134 & 140 & 151 \\
\hline 25 & 29 & $146(7)$ & 135 & 146 & 160 \\
\hline 26 & 23 & $142(7)$ & 134 & 140 & 152 \\
\hline 27 & 25 & $143(5)$ & 138 & 143 & 154 \\
\hline 28 & 17 & $145(5)$ & 138 & 145 & 158 \\
\hline 29 & 15 & $143(8)$ & 120 & 144 & 155 \\
\hline
\end{tabular}

Abbreviations: GA, gestational age; SD, standard deviation

Table 2. Fetal heart rate according to last menstrual period (LMP) during gestation

\begin{tabular}{|c|c|c|c|c|c|}
\hline GA, week & Number of cases & Mean (SD) & $5^{\text {th }}$ percentile & $50^{\text {th }}$ percentile & $95^{\text {th }}$ percentile \\
\hline 18 & 15 & $144(9)$ & 123 & 142 & 158 \\
\hline 19 & 23 & $145(6)$ & 140 & 144 & 154 \\
\hline 20 & 21 & $149(9)$ & 136 & 148 & 164 \\
\hline 21 & 34 & $144(6)$ & 135 & 145 & 156 \\
\hline 22 & 18 & $144(8)$ & 122 & 145 & 158 \\
\hline 23 & 20 & $143(9)$ & 129 & 141 & 159 \\
\hline 24 & 16 & $142(5)$ & 135 & 141 & 149 \\
\hline 25 & 23 & $144(6)$ & 138 & 140 & 153 \\
\hline 26 & 29 & $143(6)$ & 135 & 140 & 150 \\
\hline 27 & 28 & $145(6)$ & 134 & 145 & 154 \\
\hline 28 & 25 & $145(5)$ & 139 & 146 & 155 \\
\hline
\end{tabular}

Abbreviations: see Table 1

wave changes its frequency, the Doppler effect, which can be analyzed by the ultrasound apparatus to determine the speed of the object. The procedure is perfectly safe for both the mother and the fetus. Previous studies about the use of Doppler ultrasound [10] found it to be of great value in FHR monitoring, even in the $21^{\text {st }}$ century.

The FHR varies depending on the stage of pregnancy. It increases from about $100 \mathrm{bpm}$ in week 5 to $170 \mathrm{bpm}$ in week 10 . The rate is usually around $140-150 \mathrm{bpm}$ in the second trimester and 120-160 bpm in the third trimester $[11,12]$. The current international guidelines of the Fédération Internationale de Gynécologie et d'Obstétrique (FIGO) recommend a normal reference from 110 to $150 \mathrm{bpm}$; however, this value was based on the consensus from a conference in 1985 [13]. The previous consensus recommended a range of 120 to $160 \mathrm{bpm}$; this was revised on the basis of abnormal findings associated with $24 \%$ of scalp blood low analyses when FHR was higher than 160 bpm [14].

Although wider FHR ranges of 110 to $150 \mathrm{bpm}$ can be used by obstetricians during standard obstetrical ultrasound examination, more precise normal ranges related to the course of pregnancy may help to distinguish subtle abnormalities which may influence postnatal life. For example, Cuneo et al. have shown that repeated measures of $\mathrm{FHR}<3^{\text {rd }}$ percentile for gestational age or 2:1 AV conduction and/or ventricular tachycardia, diagnosed by fetal echocar- diography, were associated with long QT syndrome [15]. Even though Cuneo and her team worked with magnetocardiography, we believe that our nomograms should be used not only in research but in clinical practice as well [16].

Our results indicate that the mean value of the FHR between $18-29$ weeks is $140-145 \mathrm{bpm}$, located in the $50^{\text {th }}$ percentile.

Our tables present data from 18 to 29 weeks of pregnancy, according to fetal biometry, and 18-28 weeks of pregnancy, according to LMP. The results indicate that FHR varies according to GA: the lowest levels were noticed on week 23 and the highest values in week 20 (Figure 1). The highest observed value was $172 \mathrm{bpm}$, with a range of 30 bpm; however, this was only a temporary phenomenon related to fetal movements and otherwise, no abnormality was observed. This underlines the importance of relating the FHR with the clinical status of the fetus.

The considerable variation observed in FHR may be related to the development of the fetal heart. Its regulation depends inter alia on the function of autonomic cardiac nerves and neurotransmitters. Although the anatomical pathways appear early, the sympathetic and parasympathetic elements develop their functionality independently, with the sympathetic nervous system being formed in the first weeks of pregnancy and the parasympathetic nervous system being established later [17-19]. This difference in 
the pace of development could be the reason why lower FHR was noticed at the $25^{\text {th }}$ week of gestation, characterized by a more significant vagal tone, than in the $18^{\text {th }}$ week of gestation, with a more sympathetic tone [20]. Similarly, Nomura et al. [12] also report significant discrepancies in FHR between the second and third trimesters and propose that FHR analysis may clarify the effect of autonomic nervous system maturation on FHR regulation.

However, the present study does not examine the influences on FHR during pregnancy. It has previously been reported that the cardiac and motor development of the fetus were altered in overweight and obese mothers and that these effects became greater as the pregnancy progresses [20]. However, by 36 weeks, the fetuses of obese women showed decreased FHR variability and fewer accelerations [21, 22]. Although maternal weight before pregnancy could have influenced our results, this was not included in the data set; nevertheless, our findings represent the most recent and precise FHR nomograms for the fetal population in Poland.

Although all of the 763 cases examined in the center during the sampled period had received an umbilical Doppler blood flow assessment for FHR measurement and a pulsatility index assessment (reflecting placental resistance), the records only included FHR values for 258 fetuses (34\%). This may reflect the educational importance of our research in the future and the need to pay more attention to the value of exact FHR during gestation. FHR measurement is important for preparing an accurate prenatal medical ultrasound report. Although our fetal cardiology referral center deals with various types of fetal arrhythmia and their pharmacological treatment, FHR measurement was absent from more than $50 \%$ of cases present in the database [23-25]. Our findings demonstrate the need to improve this approach; for example, patients with FHR just below the bottom 5 th percentile should be candidates for further cardiological work-up, e.g electrocardiogram with the QTc evaluation.

Our research presents the normal ranges for FHR measured on UMB-A by ultrasound between weeks 18 and 29 of gestation in healthy fetuses with normal heart function and anatomy. These nomograms may be valuable for precise FHR assessment by prenatal and obstetrical departments in Poland and should be considered as an important element of a basic fetal ultrasound report.

\section{Article information}

Conflict of interest: SS is the author of several patents and shareholder of Medicine S.A. No products were used in this case.

Open access: This article is available in open access under Creative Common Attribution-Non-Commercial-No Derivatives 4.0 International (CC BY-NC-ND 4.0) license, allowing to download articles and share them with others as long as they credit the authors and the publisher, but without permission to change them in any way or use them commercially. For commercial use, please contact the journal office at kardiologiapolska@ptkardio.pl.
How to cite: Sylwestrzak O, Nowakowska A, Murlewska J, Respondek-Liberska M. Normal ranges of fetal heart rate values for healthy fetuses in Poland, as determined by ultrasound between weeks 18 and 29 of gestation. Kardiol Pol. 2021; 79(11): 1245-1250, doi: 10.33963/KP.a2021.0119.

\section{REFERENCES}

1. Sontakke Y. Textbook of human embryology. CBS Publishers \& Distributors, London 2018: 180-181.

2. Hamelmann $P$, Vullings R, Kolen AF, et al. Doppler ultrasound technology for fetal heart rate monitoring: a review. IEEE Trans Ultrason Ferroelectr Freq Control. 2020; 67(2): 226-238, doi: 10.1109/TUFFC.2019.2943626, indexed in Pubmed: 31562079.

3. Bhide A, Acharya G. Sex differences in fetal heart rate and variability assessed by antenatal computerized cardiotocography. Acta Obstet Gynecol Scand. 2018; 97(12): 1486-1490, doi: 10.1111/aogs.13437, indexed in Pubmed: 30091138.

4. Lim E, Miyamura J, Chen JJ. Racial/ethnic-specific reference intervals for common laboratory tests: a comparison among Asians, Blacks, Hispanics, and White. Hawaii J Med Public Health. 2015; 74(9): 302-310, indexed in Pubmed: 26468426.

5. Pildner von Steinburg $S$, Boulesteix $A L$, Lederer $C$, et al. What is the "normal" fetal heart rate? PeerJ. 2013; 1: e82, doi: 10.7717/peerj.82, indexed in Pubmed: 23761161.

6. Avitan T, Sanders A, Brain U, et al. Variations from morning to afternoon of middle cerebral and umbilical artery blood flow, and fetal heart rate variability, and fetal characteristics in the normally developing fetus. J Clin Ultrasound. 2018; 46(4): 235-240, doi: 10.1002/jcu.22569, indexed in Pubmed: 29235099.

7. Jezewski J, Wrobel J, Horoba K, et al. Monitoring of mechanical and electrical activity of fetal heart: the nature of signals. Arch Perinat Med. 2002; 8: 40-46.

8. Peters $M$, Crowe J, Piéri JF, et al. Monitoring the fetal heart non-invasively: a review of methods. J Perinat Med. 2001; 29(5): 408-416, doi: 10.1515/JPM.2001.057, indexed in Pubmed: 11723842.

9. Jezewski J, Roj D, Wrobel J, et al. A novel technique for fetal heart rate estimation from Doppler ultrasound signal. Biomed Eng Online. 2011; 10: 92, doi: 10.1186/1475-925X-10-92, indexed in Pubmed: 21999764.

10. Kupka T, Matonia A, Jezewski M, et al. New method for beat-to-beat fetal heart rate measurement using Doppler ultrasound signal. Sensors (Basel). 2020; 20(15): 4079, doi: 10.3390/s20154079, indexed in Pubmed: 32707863.

11. Pietryga M, Toboła K. Ultrasound prenatal diagnosis between 5 and 10 weeks of pregnancy [in Polish]. Gin Perinat Prakt. 2017; 2(5): 193-198.

12. Nomura RM, Campos CF, Bessa Jd, et al. Comparison of fetal heart rate patterns in the second and third trimesters of pregnancy [in Portuguese]. Rev Bras Ginecol Obstet. 2010; 32(9): 420-425, doi: 10.1590/s010072032010000900002 , indexed in Pubmed: 21271146.

13. FIGO subcommittee on Standards in Perinatal Medicine. Guidelines for the use of fetal monitoring. Int J Gynaecol Obstet. 1987; 25(3): 159-167.

14. Saling E. Das Kind im Bereich der Geburtshilfe. Georg Thieme Verlag, Stuttgart 1966.

15. Cuneo BF, Strasburger JF, Wakai RT. The natural history of fetal long QT syndrome. J Electrocardiol. 2016; 49(6): 807-813, doi: 10.1016/j.jelectrocard.2016.07.023, indexed in Pubmed: 27539165.

16. Suzin J, Respondek $A$, Respondek $M$, et al. Prenatal diagnosis of grade III congenital heart block [in Polish]. Pol Przegl Radiol. 1987; 51(1): 29-31, indexed in Pubmed: 3317299.

17. Schneider U, Schleussner E, Fiedler A, et al. Fetal heart rate variability reveals differential dynamics in the intrauterine development of the sympathetic and parasympathetic branches of the autonomic nervous system. Physiol Meas. 2009; 30(2): 215-226, doi: 10.1088/0967-3334/30/2/008, indexed in Pubmed: 19179746.

18. Schneider U, Bode F, Schmidt A, et al. Developmental milestones of the autonomic nervous system revealed via longitudinal monitoring of fetal heart rate variability. PLoS One. 2018; 13(7): e0202611, doi: 10.1371/journal.pone.0202611, indexed in Pubmed: 30106997. 
19. Jensen EC, Bennet L, Guild SJ, et al. The role of the neural sympathetic and parasympathetic systems in diurnal and sleep state-related cardiovascular rhythms in the late-gestation ovine fetus. Am J Physiol Regul Integr Comp Physiol. 2009; 297(4): R998-R1008, doi: 10.1152/ajpregu.90979.2008, indexed in Pubmed: 19641129.

20. Montalvo-Jaramillo Cl, Pliego-Carrillo AC, Peña-Castillo MÁ, et al.Comparison of fetal heart rate variability by symbolic dynamics at the third trimester of pregnancy and low-risk parturition. Heliyon. 2020; 6(3): e03485, doi: 10.1016/j.heliyon.2020.e03485, indexed in Pubmed: 32195385.

21. Voegtline KM, Costigan KA, Henderson JL, et al. Fetal heart rate and motor development in overweight and obese pregnant women. Int J Gynaecol Obstet. 2016; 133(1): 103-107, doi: 10.1016/j.ijgo.2015.08.006, indexed in Pubmed: 26797193.
22. DiPietro JA, Costigan KA, Pressman EK, et al. Antenatal origins of individual differences in heart rate. Dev Psychobiol. 2000; 37(4): 221-228, doi: 10.1002/1098-2302(2000)37:4<221::aid-dev2>3.0.co;2-a, indexed in Pubmed: 11084603.

23. Michalak A, Witczak M, Kukawczyńska E, et al. Case report and literature review. Prenatally detected non-immune atrioventricular block and maternal arrhythmia - case presentation and literature review. Prenat Cardio. 2016; 6(1): 90-95, doi: 10.1515/pcard-2016-0013.

24. Strzelecka I, Respondek-Liberska M, Słodki M, et al. Review paper. Transplacental digoxin treatment in prenatal cardiac problems in singleton pregnancies - meta analysis (based on literature: 1992-2015). Prenat Cardio. 2017; 6(1): 67-74.

25. Cuneo BF. Fetal atrial flutter and hydrops successfully treated with transplacental and direct fetal therapy. Prenat Cardio.. 2016; 6(1): 16-21. 